\title{
COVID-19 Presenting as Acute Limb Ischaemia
}

\author{
Parminder Kaur ${ }^{1}$, Sahitya Posimreddy ${ }^{1}$, Balraj Singh², Firas Qaqa ${ }^{1}$, Habib A Habib ${ }^{1}$, Michael Maroules $^{2}$, Fayez Shamoon ${ }^{1}$ \\ ${ }^{1}$ Cardiology, Saint Joseph's University Medical Center, Paterson, New Jersey, USA \\ ${ }^{2}$ Hematology \& Oncology, Saint Joseph's University Medical Center, Paterson, New Jersey, USA
}

Received: 14/05/2020

Accepted: 14/05/2020

Published: 19/05/2020

How to cite this article: Kaur P. Posimreddy S, Singh B, Qaqa F, Habib HA, Maroules M, Shamoon F. COVID-19 presenting as acute limb ischaemia. EJCRIM 2020;7: doi:10.12890/2020_001724.

Conflicts of Interests: The Authors declare that there are no competing interests.

This article is licensed under a Commons Attribution Non-Commercial 4.0 License

\section{ABSTRACT}

The severe acute respiratory syndrome coronavirus 2 (SARS-CoV-2) pandemic that developed in late 2019 and early 2020 has caused thousands of deaths and has had an enormous impact on our health systems and economies. Coronavirus disease 2019 (COVID-19) complications include disseminated coagulation and thrombosis, but, to the best of our knowledge, the literature to date on these manifestations has been limited. Herein, we report an unusual presentation in a 43-year-old man with a medical history of diabetes and hypertension who presented with dyspnoea and acute pain in his right leg and was found to have acute limb ischaemia and diabetic ketoacidosis. Our case adds to the literature regarding arterial thrombosis in COVID-19.

\section{LEARNING POINTS}

- Arterial thrombosis in the form of acute limb ischaemia can occur in COVID-19.

- A high index of suspicion should be maintained for acute limb ischaemia, which is a vascular emergency.

\section{KEYWORDS}

COVID-19, arterial thrombosis, severe acute respiratory syndrome coronavirus 2

\section{INTRODUCTION}

At the end of 2019, a novel coronavirus was identified as the source of a cluster of pneumonia cases in Wuhan, a city in Hubei province in China. Severe acute respiratory syndrome coronavirus 2 (SARS-CoV-2) causes coronavirus disease 19 (COVID-19), an extremely infectious disease mainly spread by droplets and contact $^{[1]}$. COVID-19 has provoked a global health crisis.

\section{CASE DESCRIPTION}

A 43-year-old man with a medical history of hypertension and diabetes mellitus presented to the emergency department with complaints of shortness of breath and acute right leg pain. One week previously he had started to develop fever and exertional dyspnoea. On the day of presentation, he woke up with acute pain in his right leg. Vital signs on presentation were: heart rate 130/min, blood pressure $140 / 100$ $\mathrm{mmHg}$, oxygen saturation $80 \%$ on room air, and temperature $37.1^{\circ} \mathrm{C}$. The patient had bilateral crackles on lung auscultation and an absent right dorsalis pedis pulse. His right foot was cold to the touch and mottled in appearance. Electrocardiography showed sinus tachycardia 130/min, left axis deviation, left ventricular hypertrophy with peaked T waves, and QTc $381 \mathrm{msec}$.

On initial laboratory evaluation, the following values were noted: haemoglobin $17.7 \mathrm{~g} / \mathrm{dl}$ (reference: $12-16 \mathrm{~g} / \mathrm{dl}$ ), haematocrit $59 \%$ (reference: $36-46 \%$ ), white blood cells $16 \mathrm{~K} / \mathrm{mm}^{3}$ (reference: $4.5-11 \mathrm{~K} / \mathrm{mm} 3$ ), platelets $484 \mathrm{~K} / \mathrm{mm}^{3}$ (reference: $140-440 \mathrm{~K} / \mathrm{mm}^{3}$ ), potassium $5.8 \mathrm{mEq} / \mathrm{l}$ 
(reference: $3.5-5 \mathrm{mEq} / \mathrm{l})$, blood glucose $948 \mathrm{mg} / \mathrm{dl}$ (reference: $70-105 \mathrm{mg} / \mathrm{dl}$ ), anion gap $27 \mathrm{mEq} / \mathrm{l}$ (reference: 8-16 mEq/l), small acetone, creatinine $2.74 \mathrm{mg} / \mathrm{dl}$ (reference: $0.6-1.30 \mathrm{mg} / \mathrm{dl}$ ), blood urea nitrogen $88 \mathrm{mg} / \mathrm{dl}$ (reference: $7-23 \mathrm{mg} / \mathrm{dl}$ ), lactic acid $8.7 \mathrm{mmol} / \mathrm{l}$ (reference: 0.5-2.2 mmol/l), troponin $0.497 \mathrm{ng} / \mathrm{ml}$ (reference: <0.03 ng/ml), D-dimer > 20 (reference: <0.5), prothrombin time $16.2 \mathrm{sec}$ (reference: 12.2-14.9 sec), INR 1.3 (reference: <1), partial thromboplastin time $51 \mathrm{sec}$ (reference: $21.3-35.1 \mathrm{sec}$ ), fibrinogen $853 \mathrm{mg} / \mathrm{dl}$ (reference: 183-503 mg/dl), LDH 718 U/I (reference: 140-271 U/l), CRP 289.7 mg/l (reference: <10 mg/l), ferritin 1739 ng/ml (reference: 12-300 $\mathrm{ng} / \mathrm{ml}$ ), procalcitonin $67 \mathrm{ng} / \mathrm{ml}$ (reference: <2 ng/ml), interleukin-6 $224 \mathrm{pg} / \mathrm{ml}$ (reference: 0-15.5 pg/ml), aspartate transaminase 39 U/l (reference: 13-39 U/l), calcium $8.6 \mathrm{mg} / \mathrm{dl}$ (reference: 8.6-10.3 mg/dl), and albumin $3.3 \mathrm{mg} / \mathrm{dl}$ (reference: 3.5-5.0 mg/dl).

The patient was intubated in the emergency department. Arterial blood gas analysis after intubation showed metabolic and respiratory acidosis with $\mathrm{pH}$ 6.96. A chest $\mathrm{x}$-ray showed bilateral hazy infiltrates. Computed tomography angiography of the chest, abdomen and aorta with iliofemoral run-off showed thrombus within the proximal right superficial femoral artery and absent opacification of the right popliteal artery, posterior tibial artery, peroneal artery and anterior tibial arteries compatible with occlusion (Fig. 1). It also showed extensive peripheral ground-glass infiltration of both lungs. COVID-19 was diagnosed on the basis of RT-PCR testing. The patient was placed on airborne precautions and was started on ceftriaxone, azithromycin, hydroxychloroquine and therapeutic anticoagulation with heparin. The plan was to perform percutaneous thrombectomy after correction of metabolic derangements. Diabetic ketoacidosis was managed with intravenous fluids and an insulin drip. The patient was also started on haemodialysis. Unfortunately, 2 days after admission the patient had a cardiac arrest secondary to persistent hypoxia and died.

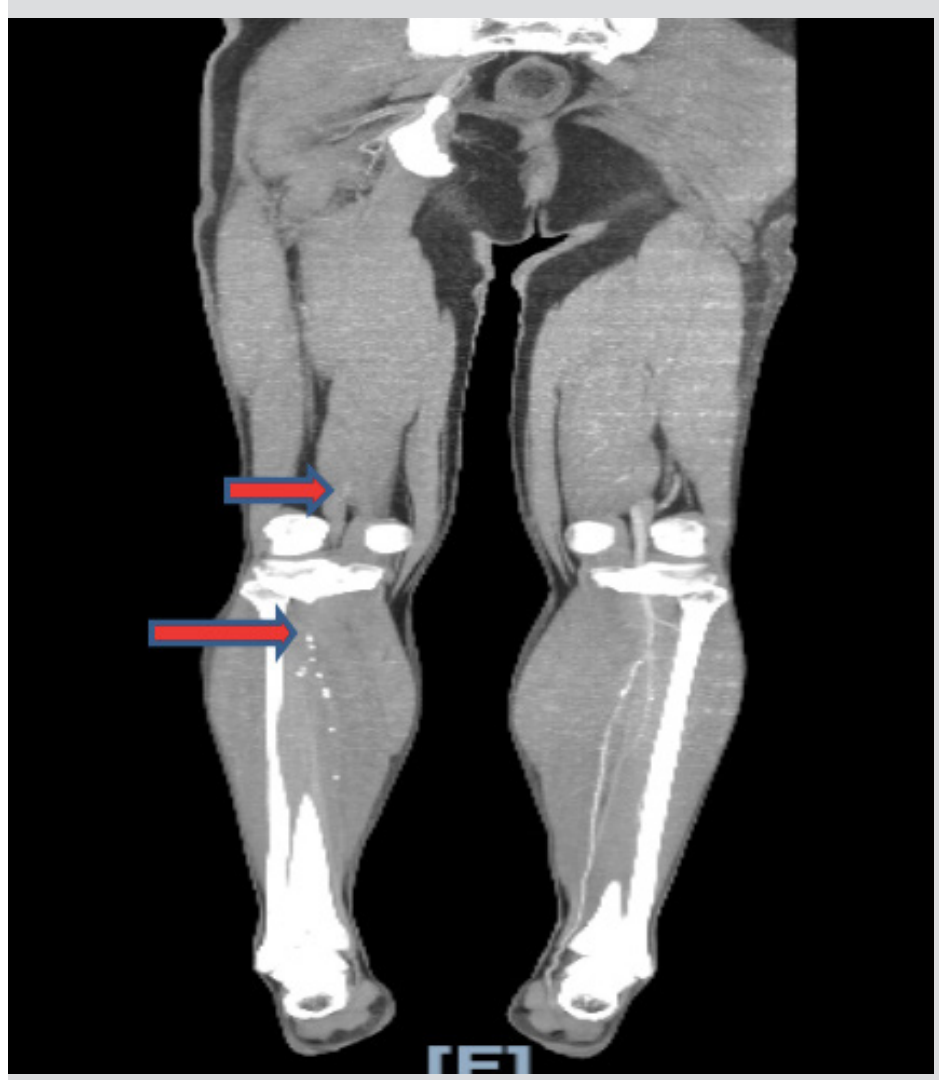

Figure 1. CT angiogram showing absent opacification of right popliteal artery, right posterior tibial artery and right peroneal artery

\section{DISCUSSION}

The COVID-19 pandemic is a fast-evolving situation. The spectrum of clinical manifestations of SARS-CoV-2 infection includes fever, myalgia, cough and dyspnoea, and less frequently headache, diarrhoea, nausea and vomiting ${ }^{[2]}$. Most infections are not severe. Of 72,314 cases reported by the Chinese Center for Disease Control and Prevention, 81\% had mild disease (no or mild pneumonia), 14\% had severe disease (e.g., dyspnoea, hypoxia, or $>50 \%$ lung involvement on imaging within 24-48 hours), and 5\% had critical disease (respiratory failure, shock, or multiorgan dysfunction) ${ }^{[3]}$. 
Patients with severe COVID-19 infection can develop disseminated intravascular coagulopathy (DIC) with fulminant activation of coagulation leading to widespread microvascular thrombosis and consumption of coagulation factors. This is reflected by thrombocytopenia, prolongation of the PT/INR and PTT[Q6], elevation of D-dimer, and decreased fibrinogen levels. In a study from Wuhan by Tang et al., 71\% of deaths from COVID-19 infection met the International Society of Thrombosis and Haemostasis (ISTH) criteria for DIC compared with $0.4 \%$ of survivors. Elevated D-dimer at admission and markedly increasing D-dimer levels (3-4-fold) over time were associated with high mortality, likely reflecting coagulation activation from infection/sepsis, cytokine storm and impending organ failure ${ }^{[4]}$.

Zhang et al. reported a similar case of a 69-year-old man with a history of hypertension, diabetes and stroke who presented with fever, cough, dyspnoea, diarrhoea and headache. COVID-19 was diagnosed on the basis of RT-PCR testing that detected SARS-CoV-2. The patient was found to have thrombosis in the lower limbs bilaterally as well as in digits two and three of the left hand and bilateral cerebral infarcts in multiple vascular territories. The same report also described two other patients with COVID-19 who had cerebral infarcts. In all three cases, serological tests were positive for anticardiolipin IgA antibodies as well as anti- $\beta 2$-glycoprotein I IgA and IgG antibodies. Lupus anticoagulant was not detected in any of the patients. These antibodies can arise transiently in patients with critical illness and various infections ${ }^{[5]}$.

The American Society of Hematology (ASH) recommends that all hospitalized patients with COVID-19 should receive pharmacological thromboprophylaxis with low-molecular-weight heparin or fondaparinux unless there is a bleeding risk and states that there is no role for full therapeutic-intensity anticoagulation unless there is an indication.

\section{CONCLUSION}

We report an unusual presentation in a 43-year-old man with a medical history of diabetes and hypertension who presented with dyspnoea and acute pain in his right leg and was found to have acute limb ischaemia and diabetic ketoacidosis. Our case and review of literature reveals that healthcare providers should be aware of the manifestations of COVID-19 and a high index of suspicion should be maintained so that all can benefit from evidence of measures that have been found to be effective.

\section{REFERENCES}

1. Jin YH, Cai L, Cheng ZS, Cheng H, Deng T, Fan YP, et al. A rapid advice guideline for the diagnosis and treatment of 2019 novel coronavirus (2019-nCoV) infected pneumonia (standard version). Mil Med Res 2020;7(1):4.

2. Guan WJ, Ni ZY, Hu Y, Liang WH, Ou CQ, He JX, et al. Clinical characteristics of coronavirus disease 2019 in China. N Engl J Med 2020;382(18):1708-1720.

3. Wu Z, McGoogan JM. Characteristics of and important lessons from the coronavirus disease 2019 (COVID-19) outbreak in China: summary of a report of 72314 cases from the Chinese Center for Disease Control and Prevention. JAMA 2020;323(13):1239-1242.

4. Tang N, Li D, Wang X, Sun Z. Abnormal coagulation parameters are associated with poor prognosis in patients with novel coronavirus pneumonia. J Thromb Haemost 2020;18(4):844-847.

5. Zhang Y, Xiao M, Zhang S, Xia P, Cao W, Jiang W, et al. Coagulopathy and antiphospholipid antibodies in patients with Covid-19. N Engl J Med 2020;382(17):e38. 